\title{
Análise temporal da fragmentação da paisagem no entorno de florestas nacionais em Rondônia
}

\author{
Fabrício Assis LEAL ${ }^{1 *}$, Francisco Ferreira dos SANTOS FILHO ${ }^{1}$, Glória da Silva Almeida LEAL ${ }^{1}$, \\ Maria Vanalda de Souza ALMEIDA ${ }^{1}$, Eder Pereira MIGUEL ${ }^{2}$
}

\author{
${ }^{1}$ Centro Multidisciplinar (CMULTI), Universidade Federal do Acre, Cruzeiro do Sul, Acre, Brasil. \\ ${ }^{2}$ Departamento de Engenharia Florestal, Universidade de Brasília, Brasília, Distrito Federal, Brasil. \\ *E-mail: fabricioassisleall@gmail.com
}

Recebido em maio/2018; Aceito em outubro/2018.

\begin{abstract}
RESUMO: O objetivo foi realizar análise temporal da fragmentação da paisagem no entorno das Florestas Nacionais do Jamari e Bom Futuro, Rondônia. Para delimitar as análises da fragmentação da paisagem foram elaboradas áreas no entorno, denominadas quadrantes (Q), nas duas Flonas: 0 a $10 \mathrm{~km}(10 \mathrm{~A}, 10 \mathrm{~B}, 10 \mathrm{C}$ e 10D) e de 10 a $20 \mathrm{~km}$ (20A, 20B, 20C e 20D). As métricas de paisagem adotadas neste trabalho foram: densidade de borda, densidade de mancha e porcentagem ocupada na paisagem. Posteriormente foram elaborados mapas com a distribuição espacial dos quadrantes que apresentaram métricas mais altas. Com a distribuição espacial dos quadrantes foi possível notar para o entorno de 0 a $10 \mathrm{~km}$ da Flona do Bom Futuro (BF), que o BF10A foi o que apresentou maior valor para duas (PD e PLAND) das três métricas utilizadas. Já para o entorno de 10 a $20 \mathrm{~km}$, apenas o quadrante BF20B se destacou entre os demais, pois para todas as métricas ele foi o mais preocupante. Com a distribuição espacial dos quadrantes foi possível notar para o entorno de 0 a $10 \mathrm{~km}$ da Flona do Jamari (JA), que o JA10C foi o que apresentou maior ocorrência para todas as métricas aqui avaliadas.

Palavras-chave: antropização, métricas de paisagem, unidade de conservação.
\end{abstract}

\section{Temporary analysis of fragmentation of the landscape in the environment of national forests}

\begin{abstract}
The objective was to carry out a temporal analysis of the fragmentation of the landscape around the National Forests of Jamari and Bom Futuro, Rondônia. In order to delimit the analyzes of the landscape fragmentation, areas in the surroundings, called quadrants $(\mathrm{Q})$, were elaborated in the two Flonas: 0 to $10 \mathrm{~km}$ $(10 \mathrm{~A}, 10 \mathrm{~B}, 10 \mathrm{C}$ and $10 \mathrm{D})$ and 10 to $20 \mathrm{~km}(20 \mathrm{~A}, 20 \mathrm{~B}, 20 \mathrm{C}$ and 20D). The landscape metrics adopted in this work were: border density, spot density and percentage occupied in the landscape. Subsequently maps were elaborated with the spatial distribution of the quadrants that presented higher metrics. With the spatial distribution of the quadrants, it was possible to note that the BF10A was the one with the highest value for two (PD and PLAND) of the three metrics used for the 0 to $10 \mathrm{~km}$ environment of the Bom Futuro Flona (BF). Already for the environment of 10 to $20 \mathrm{~km}$, only the quadrant BF20B stood out among the others, because for all the metrics he was the most worrisome. With the spatial distribution of the quadrants it was possible to notice for the environment of 0 to $10 \mathrm{~km}$ of Jamari Flona (JA), that the JA10C was the one that presented the highest occurrence for all the metrics evaluated here.
\end{abstract}

Keywords: anthropization process, landscape metrics, conservation unit.

\section{INTRODUÇÃO}

Até os anos 70, antes do programa de ocupação da Amazônia, ainda existiam florestas sem intervenção antrópica, mas desde então, essas grandes áreas de florestas sofreram perdas arbitrárias, marcando atualmente uma área conhecida como "arco do desmatamento", que se estende desde Maranhão até Rondônia. Esta área recebe essa denominação devido ao alto índice de desmatamento da Floresta Amazônica (16\%), resultante da perda de mais de $676.000 \mathrm{~km}^{2}$ de florestas nativas (SILVA et al., 2016).

Segundo dados do INPE (2018) de monitoramento da Floresta Amazônica, a região da Amazônia Legal apresentou quedas de desflorestamento de 2004 até 2012, com registro de área desmatada mínima de $4.571 \mathrm{~km}^{2}$, em 2012. Desde então houve aumento novamente da área desmatada, como pode ser verificado no ano de $2016 \mathrm{com} 7.893 \mathrm{~km}^{2}$, chegando nos atuais $6.624 \mathrm{~km}^{2}$ em 2017. Rondônia, um dos três estados que mais desmataram na Amazônia Legal nesse mesmo período (após 2004), apresentou esse mesmo padrão com ligeiras quedas e o menor registro de desmatamento em $2010\left(435 \mathrm{~km}^{2}\right)$, porém, houve crescimento expressivo de desmatamento, chegando ao ano de $2017 \mathrm{com} 1.252 \mathrm{~km}^{2}$. Atualmente o estado representa 13,8\% do desmatamento ocorrido na Amazônia Legal, com $59.113 \mathrm{~km}^{2}$ desmatados (INPE, 2018).

Nas últimas quatro décadas, a principal característica da ocupação da Amazônia, pelo colonizador, foi a destruição do componente mais caracteristicamente amazônico do bioma: a floresta. Exclusivamente, não há registros na história humana de algum povo que tenha destruído com tanta atrocidade e celeridade um patrimônio botânico como esse, em grande parte, perdido para sempre (SCHMINK, 2012).

Diante deste cenário, uma maneira para conter 0 desmatamento e preservar florestas remanescentes na Amazônia Legal e em outros locais do país foi a criação de 65 
Florestas Nacionais (FLONA) no Brasil (BASTOS et al. 2014), que são áreas protegidas instituídas pelo Sistema Nacional de Unidade de Conservação (SNUC) Lei $n^{\circ}$ 9.985/2000, se enquadrando no tipo Uso Sustentável. Três delas estão no estado de Rondônia, sendo: FLONA do Bom Futuro, do Jamari e de Jacundá. Destacam-se entre elas as FLONAS do Bom Futuro e do Jamari, pelas grandes intervenções antrópicas no entorno das mesmas, causando fragmentação pelo alto índice de desmatamento, presença de áreas de pastagens, agricultura e garimpo. A FLONA do Bom Futuro foi criada pelo decreto $\mathrm{n}^{\circ} 96.188$, de 21 de junho de 1988 e a FLONA do Jamari foi criada pelo decreto $n^{\circ} 90.224$, de 25 de setembro de 1984.

Uma forma de controlar o avanço da antropização nos arredores das áreas protegidas, o Sistema Nacional de Unidade de Conservação (SNUC) garantiu em lei limites de proteção para espécies vegetais e animais, criando critérios de exploração e acompanhamento nas zonas de amortecimento, a fim de barrar os avanços sobre as demarcações das áreas protegidas, minimizando as pressões sobre o espaço tampão, se tornando um espaço essencial para propagação e proteção da biodiversidade (RIBEIRO et al., 2010).

Entretanto, tais medidas não foram capazes de frear o avanço da antropização nas zonas de amortecimento, e os resultados destas consequências são ambientes cada vez mais fragmentados e carentes de estudos para melhor compreensão. Para Couto (2004) e Silva (2014), métricas da paisagem também conhecidas por índices da paisagem, são instrumentos úteis e essenciais para descrever, comparar e quantificar modelos espaciais de fragmentos de florestas nativas.

A adoção desses índices permite gerar dados, informações envolvendo manchas de florestas, seja individual ou coletiva, construindo uma base computacional para gerar definições da paisagem, da mancha de floresta e estudar a conectividade e configuração da paisagem. Sendo assim, é uma maneira de representar de forma sucinta aspectos sobre uma área de floresta fragmentada e fornecer informações úteis que possam ser utilizadas na gestão de zonas de amortecimento.

Diante deste contexto, o objetivo deste trabalho foi realizar análise temporal da fragmentação da paisagem em áreas de entorno das Florestas Nacionais do Jamari e do Bom Futuro/RO, em 2004 e 2014, por meio de índices de paisagem.

\section{MATERIAL E MÉTODOS}

\subsection{Localização da área de estudo}

O estudo contemplou duas Florestas Nacionais no estado de Rondônia, estado esse que se localiza na região Norte do Brasil, onde ocupa área geográfica de $237.576 \mathrm{~km}^{2}$ (FIORI et al., 2013). O estudo foi realizado em duas de suas FLONAS: do Bom Futuro e do Jamari. A primeira tem área de 97.385 ha, está localizada no município de Porto Velho, e a FLONA do Jamari, com área de aproximadamente 215.000 ha, abrange os municípios de Cujubim, Candeias do Jamari e Itapuã do Oeste (Figura 1).

\subsection{Base de dados, elaboração e compartimentação das áreas de entorno}

Para avaliar a fragmentação da paisagem foram utilizados os dados vetoriais (shapefile) do projeto TerraClass em $2004 \mathrm{e}$ 2014, respectivamente, o primeiro e o último dado disponível contendo o mapeamento do uso e cobertura da terra na Amazônia Legal brasileira (ALMEIDA et al., 2016).

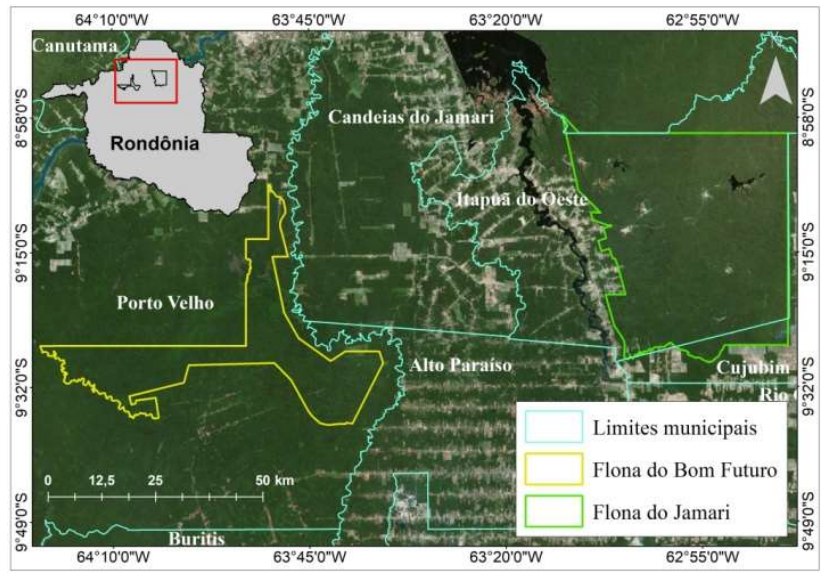

Figura 1. Localização da área de estudo envolvendo as Florestas Nacionais do Bom Futuro (amarelo) e do Jamari (verde), no estado de Rondônia. Fonte: Malha municipal digital do IBGE e imagem BaseMap ESRI.

Figure 1. Location of the study area as Bom Futuro National Forests (yellow) and Jamari (green), in the state of Rondônia. Source: Malha municipal digital do IBGE and image BaseMap ESRI.

Os dados do TerraClass foram baixados de acordo com a articulação das cenas do satélite Landsat (órbita/ponto). As cenas baixadas foram: 233/66, 233/67, 232/66, 232/67, 231/66 e $231 / 67$, ao todo foram baixadas seis cenas do mapeamento para cada ano avaliado (2004 e 2014).

Para análise da fragmentação da paisagem foram elaboradas áreas no entorno das FLONAS do Bom Futuro e do Jamari. A primeira área de entorno foi definida conforme limites das zonas de amortecimento de $0-10 \mathrm{~km}$ prevista no SNUC. Nessa área foi avaliada a fragmentação da paisagem das áreas adjacentes às florestas nacionais (mais próximo). A segunda área de entorno, também para análise da fragmentação, foi definida num raio de $10-20 \mathrm{~km}$ do limite de cada uma das unidades de conservação e serviram para avaliar a região externa adjacente às zonas de amortecimento. Perfazendo-se assim, duas áreas de análise para cada FLONA.

Para entender melhor a fragmentação da paisagem na área de estudo, as áreas de entorno supracitadas foram compartimentadas em quadrantes (Q). Esses quadrantes serviram para avaliar a fragmentação da paisagem de forma direcional, e foram avaliados tanto para 2004 como para 2014. Cada quadrante foi renomeado e isolado em arquivos individuais (shapefile). A área de entorno de 0-10 km seus quadrantes receberam os nomes de 10A, 10B, 10C, 10D, e a área de entorno de 10-20 km seus quadrantes receberam os nomes de 20A, 20B, 20C e 20D, ordenados no sentido horário e precedidos das iniciais BF (Bom Futuro) e JA (Jamari).

\subsection{Conversão dos dados do TerraClass}

Os arquivos vetoriais do TerraClass, tanto para 2004 como para 2014, foram convertidos para o formato raster (.tif) objetivando facilitar os processos de análise da fragmentação. $\mathrm{O}$ arquivo raster gerado foi então recortado conforme cada quadrante avaliado. Os quadrantes foram submetidos à análise da fragmentação por meio do software FRAGSTAT 4.2 (PEREIRA et al., 2012; (MCGARIGAL et al., 2012; 1995).

Nesta abordagem a classe de uso do solo do TerraClass prioritária para análise da fragmentação foi a classe "floresta", sendo as demais não consideradas na análise de fragmentação da paisagem. 


\subsection{Análise da fragmentação da paisagem}

Neste tópico buscou-se mensurar e comparar o grau de fragmentação dos remanescentes de vegetação nativa (classe "floresta" do TerraClass), por meio de índices de paisagem. Os índices utilizados se enquadram na categoria "classe" e foram escolhidos por serem amplamente utilizados em análises de paisagem (OLIVEIRA et al., 2015; PIROVANI et al., 2014; FRANÇOSO; BRANDÃO, 2013; VIDOLIN et al., 2011) por apresentarem suas unidades relativizadas por unidades de área, sendo eles:

- Densidade de borda (ED) (Equação 1), cuja unidade é $\mathrm{m} / \mathrm{ha}$, que significa a quantidade de extremidade relativa à área da paisagem.

$$
E D=\frac{\sum_{k=1}^{m \prime} e i k}{A}(10.000)
$$

(Equação 1)

em que: $\mathrm{k}=1 ; \mathrm{m}$ ' = tipos de manchas (classes); $\mathrm{e}_{\mathrm{ik}}=$ comp. total (m) de borda na paisagem entre os tipos de manchas(classes) i e $\mathrm{k} ; \mathrm{A}=$ área total da paisagem

- Densidade de mancha (PD) (Equação 2), cuja unidade é mancha $/ \mathrm{km}^{2}$, que expressa o número de mancha de uma determinada classe da paisagem por unidade de área.

$$
P D=\frac{N}{A}(10.000) x(100) \quad \text { (Equação 2) }
$$

em que: $\mathrm{N}$ = quantidade de mancha na paisagem; $\mathrm{A}=$ área total da paisagem.

- Porcentagem da mancha (PLAND), cuja unidade é porcentagem (\%), que representa a proporção da paisagem ocupada por determinada mancha.

$$
\text { PLAND }=P_{i}=\frac{\sum_{j=1}^{n} a i j}{A}(100)
$$

(Equação 3)

em que: $P i=$ proporção da paisagem ocupada por um tipo de mancha (classe) $i$; aij = área da mancha $\left(\mathrm{m}^{2}\right) ; \mathrm{A}=$ área total da paisagem $\left(\mathrm{m}^{2}\right)$.

Ao fim dos cálculos dos índices, os resultados foram comparados entre si, e posteriormente foram elaborados mapas com a distribuição espacial de cada quadrante com o seu respectivo índice de paisagem.

\section{RESULTADOS}

3.1. Áreas de entorno compartimentadas das Florestas Nacionais do Jamari e do Bom Futuro

A Figura 2 ilustra o resultado obtido após a elaboração das áreas de entorno de ambas as FLONAS. Flona do Jamari: cor verde escura representa a área de entorno de 0 a $10 \mathrm{~km}$ (subdividida: JA10A, JA10B, JA10C e JA10D) e a cor verde claro representa a área de entorno de 10 a $20 \mathrm{~km}$ (subdividida: JA20A, JA20B, JA20C e JA20D). Flona do Bom Futuro: cor azul escuro consiste na representação da área de entorno de 0 a $10 \mathrm{~km}$ (subdividida: BF10A, BF10B, BF10C e BF10D) e a cor azul claro de 10 a $20 \mathrm{~km}$ (subdividida: BF20A, BF20B, BF20C e BF20D).

Após a compartimentação das áreas de entorno obteve-se os cenários para avaliação e comparação da fragmentação da paisagem em 2004 e 2014. Os cenários para comparação foram distintos, ou seja, não houve comparação entre as duas FLONAS, apenas entre os quadrantes na mesma Flona (2004 e 2014)

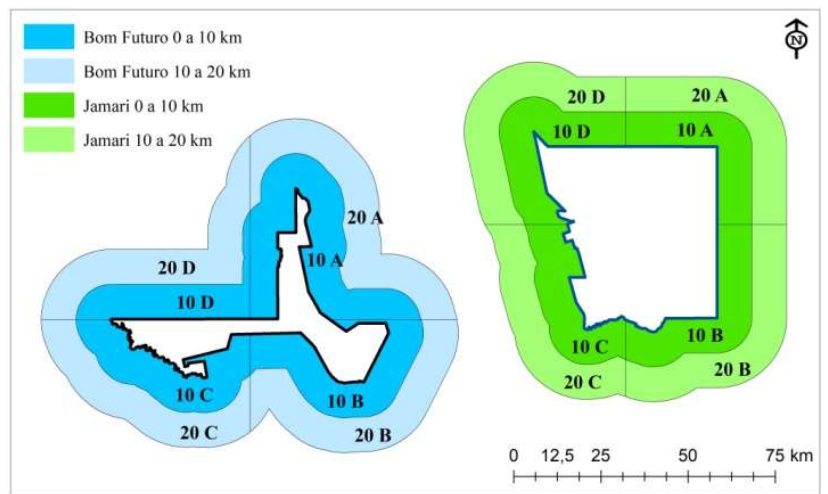

Figura 2. Áreas compartimentadas do entorno das FLONAS do Jamari e do Bom Futuro, bem como a identificação de cada um de seus quadrantes.

Figure 2. Compartmentalized areas of the surroundings of the Jamari FLONAS and the Bom Futuro, as well as the identification of each of its quadrants.

\subsection{Dados do TerraClass utilizados na análise}

A Figura 3 ilustra o arquivo raster gerado após conversão dos dados do TerraClass para o ano de 2004. Percebe-se que ambas as FLONAS estão circundadas por áreas com importante pressão antrópica.

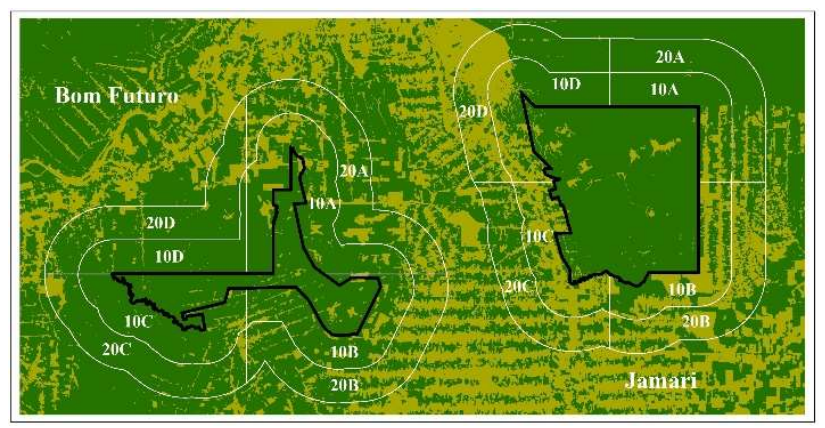

Figura 3. Raster gerado após a conversão dos dados do TerraClass de 2004 para avaliar a fragmentação da paisagem, nas FLONAS do Bom Futuro e do Jamari. A classe "floresta" foi representada pela cor verde e a cor amarela representou as áreas antropizadas.

Figure 3. Raster generated after the conversion of TerraClass data from 2004 to evaluate the fragmentation of the landscape, in the Flonas of Bom Futuro and Jamari. The forest class was represented by green color and yellow color as the anthropized areas.

É possível notar, na Figura 3, que em 2004 as áreas de entorno das duas FLONAS já apresentavam antropização considerável, bem como no interior de ambas. A frequente ação do homem em explorar seus recursos naturais, tanto no interior das FLONAS como em seu entorno, causou conflitos entre posseiros e agentes do Instituto Brasileiro do Meio Ambiente e dos Recursos Renováveis (IBAMA).

O pico da ocupação foi por volta de 2010, iniciando-se ao longo da BR-364, à medida que a ocupação da margem foi adentrando e desmatando as unidades. Tal ocupação se deu para retirada de produto madeireiro e mineral como cassiterita, somados com a criação de gado extensiva. Na Flona do Jamari, por exemplo, na área de entorno 10 a $20 \mathrm{~km}$ ocorre, visualmente, maior presença de área antropizada onde se localizam os quadrantes JA20B e JA20C. Já na área de entorno de 0 a $10 \mathrm{~km}$ chamam a atenção aos quadrantes JA10B e JA10C, pela ausência quase total de vegetação nativa. Em relação aos compartimentos um pouco menos antropizados destacam os quadrantes JA10A e JA10D e JA20A e JA20D. 
Em resumo, a parte Sul da área de entorno da Flona do Jamari sofreu maior influência antrópica até 2004.

Já a Flona do Bom Futuro mostra que os quadrantes BF20A, BF20B, BF10A e BF10B são aqueles que apresentaram maior área antropizada até 2004, os demais quadrantes, BF10C, BF10D, BF20C e BF20D foram os que apresentaram maior área para a classe "florestas". Em linhas gerais, a área leste da Flona do Bom Futuro sofreu até o ano 2004 maior influência antrópica, e a área oeste da mesma Flona menor impacto antrópico. Obviamente há variáveis que podem explicar tais acontecimentos de tal área ser mais desmatada que outras, por exemplo, a declividade pode ter afetado determinadas áreas impossibilitando o desmatamento em alguns locais dos quadrantes. Entretanto, as análises para quantificar a fragmentação da paisagem, aqui sugeridas, não conseguem considerar isso.

Para o ano de 2014, representado na Figura 4 pode ser visualizado o arquivo raster gerado após conversão dos dados do TerraClass para o ano de 2014. Percebe-se que ambas as FLONAS estão circundadas por áreas com importante pressão antrópica. Foi notório o avanço das áreas antropizadas sobre as áreas com vegetação nativa de 2004 (Figura 3) para 2014 (Figura 4). Poucos quadrantes ficaram como estavam em 2004, porém a grande maioria teve aumento importante das áreas antropizadas.

$\mathrm{O}$ fato que chama a atenção é na parte norte na Flona do Jamari onde (quadrantes JA10A e JA20A) pouco se percebeu em relação ao aumento das áreas antropizadas. Conforme verificado por meio de arquivos de unidades de conservação e terras indígenas, não existe nenhuma dessas unidades de conservação naquela porção da área de estudo.

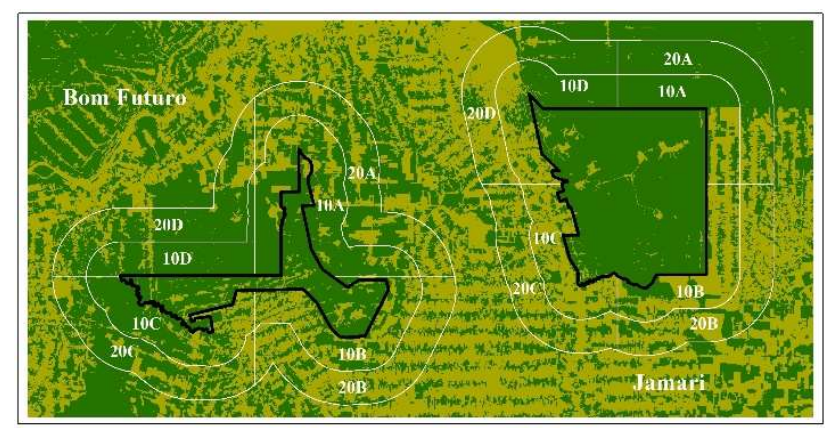

Figura 4. Raster gerado após a conversão dos dados do TerraClass de 2014 para avaliar a fragmentação da paisagem, nas FLONAS do Bom Futuro e do Jamari.

Figure 4. Raster generated after the conversion of TerraClass data from 2014 to evaluate the fragmentation of the landscape, in the Flonas of Bom Futuro and Jamari.

Em linhas gerais sobre a avaliação visual percebe-se que houve bastante avanço das áreas antropizadas após 10 anos (2004 a 2014), isso significa dizer que a fragmentação da paisagem também sofreu avanços, refletindo em fragmentos mais isolados. Tal fato foi evidenciado nos valores das métricas de paisagem encontrados nesta avaliação.

\subsection{Análise da paisagem}

Para facilitar o entendimento sobre as análises e comparações da paisagem aqui propostas, os resultados das métricas foram apresentados por FLONAS. Os resultados para cada Flona foram apresentados em dois conjuntos de gráficos (0 a $10 \mathrm{~km}$ e 10 a $20 \mathrm{~km}$ ), em que cada conjunto de gráficos reuniu as três métricas aqui consideradas (PD, ED e PLAND), bem como os dois períodos avaliados (2004 e 2014).

\subsubsection{Floresta Nacional do Bom Futuro}

Para a Flona do Bom Futuro o primeiro conjunto de gráficos com as comparações foi para a área de entorno de $0 \mathrm{a}$ $10 \mathrm{~km}$, assim sendo, as métricas da paisagem PD, ED e PLAND para seus respectivos quadrantes podem ser visualizadas na Figura 5.
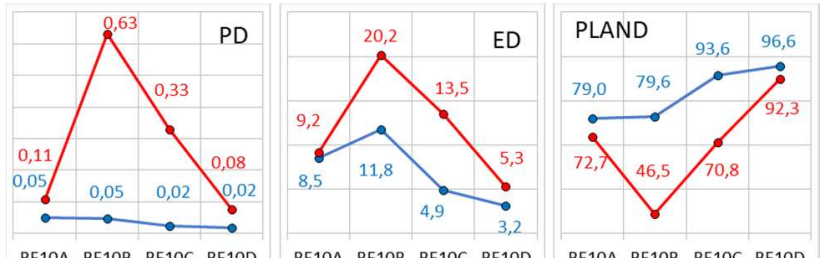

BF10A BF10B BF10C BF10D BF10A BF10B BF10C BF10D BF10A BF10B BF10C BF10D Figura 5. Métricas da paisagem PD, ED e PLAND da área de entorno de 0 a $10 \mathrm{~km}$, em seus respectivos quadrantes para a Flona do Bom Futuro. A cor azul das linhas se refere ao ano de 2004 e a cor vermelha ao ano de 2014.

Figure 5. PD, ED and PLAND landscape metrics of the surrounding area from 0 to $10 \mathrm{~km}$, in their respective quadrants for the Bom Futuro Flona. The blue color of the lines refers to the year 2004 and the color red to the year 2014.

Conforme pode ser observado na Figura 5, a densidade de mancha (PD) aumentou em todos os quadrantes, sendo as maiores diferenças nos BF10B de 0,05 para 0,63 mancha $/ \mathrm{km}^{2}$ e no $\mathrm{BF} 10 \mathrm{C}$ de 0,02 para 0,33 mancha $/ \mathrm{km}^{2}$. Tal fato foi comprovado pelo aumento no total de bordas (ED) no BF10B de 11,8 para $20,2 \mathrm{~m} /$ ha e no BF10C de 4,9 para $13,5 \mathrm{~m} / \mathrm{ha}$ em 2014. Em relação a porcentagem da paisagem ocupada pela mancha (PLAND), os quadrantes BF10A e BF10D foram os únicos que perderam pouca vegetação nativa em dez anos no entorno de 0 a $10 \mathrm{~km}$, respectivamente, de $79,0 \%$ para $72,7 \%$ $(6,3 \mathrm{pp})$ e de $96,6 \%$ para $92,3 \%(4,3 \mathrm{pp})$.

Para os outros dois quadrantes (BF10C e BF10B) houve intensa alteração na paisagem nesse intervalo de 10 anos (BF10C de 93,6\% para 70,8\% e BF10B de 79,6\% para 46,5\%). Toda essa alteração antrópica foi refletida no quadrante BF10B, que foi o que apresentou maior densidade de bordas $(0,63 \mathrm{~m} / \mathrm{ha})$ entre todos. Este valor significa que os fragmentos contidos neste quadrante são mais irregulares, por isso apresentaram mais densidade de bordas que os demais.

O segundo conjunto de gráficos, com as comparações para a Flona do Bom Futuro, foi para a área de entorno de 10 a 20 $\mathrm{km}$. Da mesma forma que o anterior, as métricas da paisagem PD, ED e PLAND para seus respectivos quadrantes podem ser visualizadas na Figura 6.
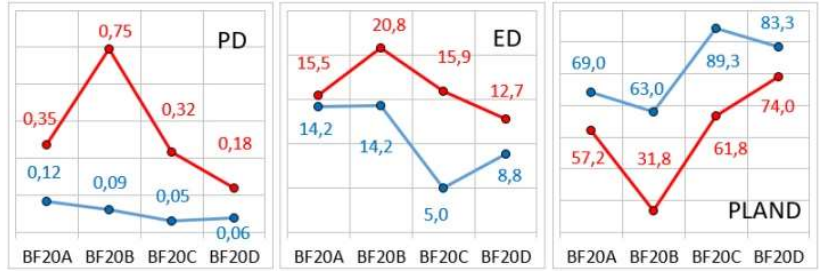

Figura 6. Métricas da paisagem PD, ED e PLAND da área de entorno de 10 a $20 \mathrm{~km}$, em seus respectivos quadrantes para a Flona do Bom Futuro. A cor azul das linhas se refere ao ano de 2004 e a cor vermelha ao ano de 2014.

Figure 6. PD, ED and PLAND landscape metrics of the surrounding area from 10 to $20 \mathrm{~km}$, in their respective quadrants for the Bom 
Futuro Flona. The blue color of the lines refers to the year 2004 and the color red to the year 2014 .

Conforme pode ser observado na Figura 6, todos os quadrantes de 10 a $20 \mathrm{~km}$ apresentaram alterações na paisagem em dez anos, haja visto que as métricas não repetiram valores para o ano de 2014 (linha vermelha). O quadrante BF20B foi onde mais ocorreu antropização, pois em 2004 havia 63,0\% de vegetação nativa (PLAND) e em 2014 restaram apenas 31,8\%, uma queda considerável que refletiu nas outras métricas. Se observarmos é fácil constatar que a densidade de mancha (PD) para BF20B saiu de 0,09 em 2004 para 0,75 mancha $/ \mathrm{km}^{2}$ em 2014. Logo, tais consequências resultaram no aumento de densidade de borda (ED), passando de 14,2 para 20,8 m/ha em 2014.

O quadrante BF20C também apresentou alterações notáveis em dez anos. Em termos de pontos percentuais (pp), este quadrante foi o segundo que perdeu mais vegetação nativa, passando de $89,3 \%$ para $61,8 \%$ (PLAND). Este resultado também refletiu nas outras métricas, em que houve aumento na densidade de borda (ED), de 5,0 para $15,9 \mathrm{~m} / \mathrm{ha}$ em 2014, bem como na densidade de manchas (PD), de 0,05 para 0,32 mancha $/ \mathrm{km}^{2}$, evidenciando aumento na fragmentação da paisagem.

Para finalizar as análises na Flona do Bom Futuro deve-se ressaltar que para o entorno de 0 a $10 \mathrm{~km}$, o quadrante com o pior cenário entre todos para todas as métricas foi o $\mathrm{BF} 10 \mathrm{~B}$, pois apresentou os maiores valores para as métricas $(\mathrm{PD}=0,63$ mancha $/ \mathrm{km}^{2}, E D=20,2 \mathrm{~m} / \mathrm{ha}$, PLAND $=46,5 \%$ ). Para o entorno de 10 a $20 \mathrm{~km}$, o quadrante BF20B concentrou os piores cenários para todas as métricas $\left(\mathrm{PD}=0,75 \mathrm{mancha} / \mathrm{km}^{2}\right.$, $\mathrm{ED}=20,8 \mathrm{~m} / \mathrm{ha}, \mathrm{PLAND}=31,8 \%$ ). Estes fatos evidenciam que a região sudeste da Flona do Bom Futuro é a que foi mais atingida pela antropização.

A distribuição espacial dos quadrantes que apresentaram, comparativamente, as métricas mais altas que os demais, pode ser visualizado na Figura 7.

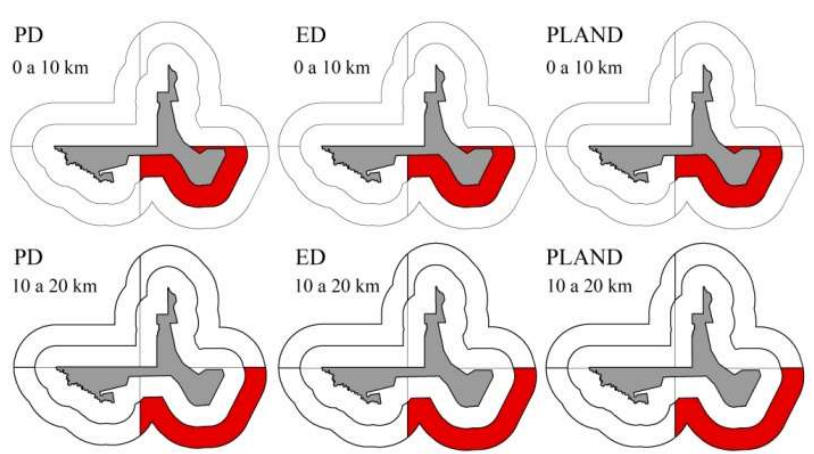

Figura 7. Distribuição espacial dos quadrantes que apresentaram valores de métricas mais relevantes que os demais, para PD, ED e PLAND, no entorno da Flona do Bom Futuro.

Figure 7. Spatial distribution of the quadrants that presented values of metrics more relevant than the others, for PD, ED and PLAND, in the surroundings of Flona Bom Futuro.

Com a distribuição espacial dos quadrantes (Figura 7) é possível notar para o entorno de 0 a $10 \mathrm{~km}$, que o quadrante $\mathrm{BF} 10 \mathrm{~B}$ foi o que apresentou os maiores valores para todas as métricas utilizadas (PD, ED e PLAND). Este quadrante, em especial, se torna essencial para estudos e acompanhamentos da evolução do uso do solo, pois reúne condições de relevante interesse para mitigar as consequências da antropização. Já para o entorno de 10 a $20 \mathrm{~km}$, apenas o quadrante BF20B se destacou entre os demais, pois para todas as métricas ele foi o mais preocupante e merece atenção e monitoramento para essa antropização não avance para o entorno de 0 a $10 \mathrm{~km}$ e fique mais próximo ainda da Flona do Bom Futuro.

\subsubsection{Floresta Nacional do Jamari}

Para a Flona do Jamari o primeiro conjunto de gráficos com as comparações foi para a área de entorno de 0 a $10 \mathrm{~km}$, assim sendo, as métricas da paisagem PD, ED e PLAND para seus respectivos quadrantes podem ser visualizadas na Figura 8.

Percebe-se que houve aumento na densidade de manchas (PD) em todos os quadrantes analisados. Em especial o JA10C e o JA10D foram os que mais apresentaram aumento no período avaliado, respectivamente, de 0,82 para 1,14 mancha $/ \mathrm{km}^{2}$ e de 0,67 para 0,87 mancha $/ \mathrm{km}^{2}$. Já em relação ao comprimento de bordas (ED), embora a densidade de manchas (PD) tenha aumentado em todos os quadrantes, essa métrica não apresentou mudanças notáveis. Pelo contrário, as métricas de 2004 para 2014 mudaram muito pouco, o que significa dizer que embora tenha ocorrido antropização nos quadrantes, este acontecimento não influenciou muito na forma dos fragmentos remanescentes. Em relação a proporção de ocupação na paisagem (PLAND), por determinada classe, mudanças foram notadas e, em alguns quadrantes, quase ou mais de 10pp (pontos percentuais) foram convertidos em áreas antropizadas em 2014. São os casos do JA10A, JA10B e JA10C, respectivamente, de $85,3 \%$ para $75,2 \%(10,1 \mathrm{pp})$, de $45,2 \%$ para $35,6 \%(9,6 p p)$ e de $38,4 \%$ para $29,4 \%(9 p p)$.

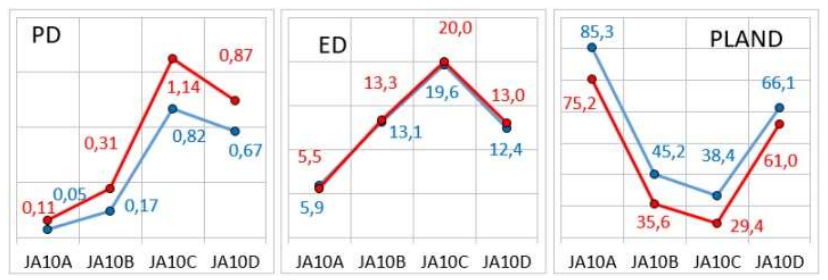

Figura 8. Métricas da paisagem PD, ED e PLAND da área de entorno de 0 a $10 \mathrm{~km}$, em seus respectivos quadrantes para a Flona do Jamari. A cor azul das linhas se refere ao ano de 2004 e a cor vermelha ao ano de 2014.

Figure 8. PD, ED and PLAND landscape metrics of the surrounding area from 0 to $10 \mathrm{~km}$, in their respective quadrants for the Jamari Flona. The blue color of the lines refers to the year 2004 and the color red to the year 2014 .

O segundo conjunto de gráficos, com as comparações para a Flona do Jamari, foi para a área de entorno de 10 a $20 \mathrm{~km}$. Da mesma forma que o anterior, as métricas da paisagem PD, ED e PLAND para seus respectivos quadrantes podem ser visualizadas na Figura 9.

Após avaliar a Figura 9 percebe-se que todos tiveram suas métricas alteradas no período de 10 anos. Entretanto, apenas alguns quadrantes foram mais visíveis essas alterações. No caso da densidade de mancha (PD), o quadrante JA20C foi o que mais apresentou contraste entre o período analisado, pois passou de 0,22 para 0,62 mancha/ $\mathrm{km}^{2}$ (aumento de 0,40 mancha $/ \mathrm{km}^{2}$ ). Os quadrantes JA20B e JA20D também apresentaram aumento para densidade de manchas, respectivamente, de 0,23 para 0,48 mancha $/ \mathrm{km}^{2}$ (aumento de $0,25 \mathrm{mancha} / \mathrm{km}^{2}$ ) e de 0,86 para 1,04 mancha $/ \mathrm{km}^{2}$ (aumento de 0,18 mancha $/ \mathrm{km}^{2}$ ). 


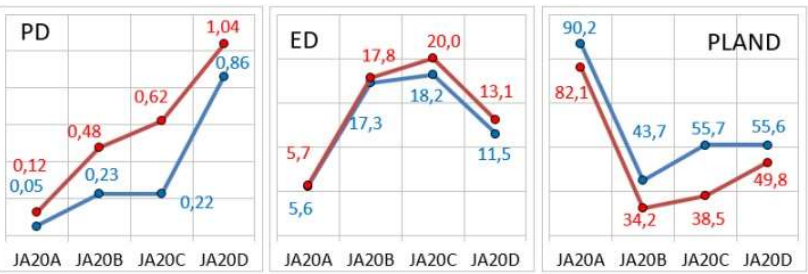

Figura 9. Métricas da paisagem PD, ED e PLAND da área de entorno de 10 a $20 \mathrm{~km}$, em seus respectivos quadrantes para a Flona do Jamari A cor azul das linhas se refere ao ano de 2004 e a cor vermelha ao ano de 2014

Figure 9. PD, ED and PLAND landscape metrics of the surrounding area from 10 to $20 \mathrm{~km}$, in their respective quadrants for the Jamari Flona. The blue color of the lines refers to the year 2004 and the color red to the year 2014

A métrica densidade de bordas (ED) não apresentou resultados muito diferentes daqueles encontrados em 2004, porém os quadrantes JA20C e JA20D foram os mais representativos, pois aumentaram respectivamente 1,8 e 1,6 $\mathrm{m} / \mathrm{ha}$ em 10 anos. A métrica que explica a ocupação de paisagem (PLAND) foi possível notar que o quadrante mais preservado foi o JA20A, mesmo ainda que ele tenha saído de $90,2 \%$ para $82,1 \%(8,1 \mathrm{pp})$.

Os outros três quadrantes (JA20B, JA20C e JA20D) já apresentam menos que $50 \%$ de vegetação nativa. Chama a atenção o JA20B que passou de $43,7 \%$ para apenas $34,2 \%$, o JA20C de $55,7 \%$ passou a ter apenas $38,5 \%$ e o JA20D que de $55,6 \%$ passou a ter $49,8 \%$. A distribuição espacial dos quadrantes que apresentaram, comparativamente, métricas mais relevantes que os demais, pode ser visualizado na Figura 10 .
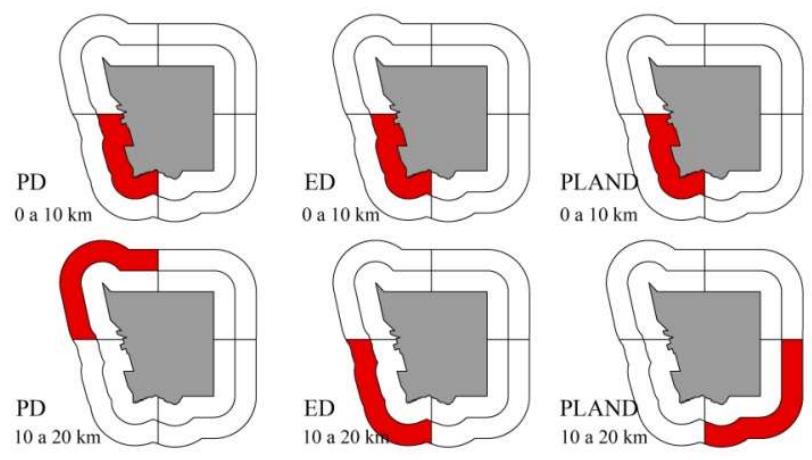

Figura 10. Distribuição espacial dos quadrantes que apresentaram valores de métricas mais relevantes que os demais, para PD, ED e PLAND, no entorno da Flona do Jamari.

Figure 10. Spatial distribution of the quadrants that presented values of metrics more relevant than the others, for PD, ED and PLAND, in the surroundings of Flona Jamari.

Com a distribuição espacial dos quadrantes acima foi possível notar para o entorno de 0 a $10 \mathrm{~km}$, que o JA10C foi o que apresentou maior ocorrência para todas as métricas aqui avaliadas. Este quadrante requer atenção espacial, pois foi o mais preocupante entre os cenários avaliados e também por estar na região da zona de amortecimento da unidade de conservação. Já para o entorno de 10 a $20 \mathrm{~km}$, três foram os quadrantes que se destacaram, sendo o JA20D para a métrica densidade de mancha (PD), ou seja, é o mais fragmentado entre os outros. O JA20C apresentou maior densidade de borda (ED), o que quer dizer que os fragmentos são os mais irregulares e o JA20B que foi aquele com menor quantidade de vegetação nativa remanescente entre os outros.

\section{DISCUSSÃO}

Vários foram os trabalhos que envolveram métricas para quantificar a estrutura da paisagem de forma satisfatória. O trabalho de Cruz et al. (2013), foi assertivo ao incluir as métricas, número de mancha (PD) e densidade de bordas (ED) para analisar a fragmentação da paisagem, no noroeste de Portugal. Nesta abordagem, as métricas utilizadas foram capazes de medir o grau de fragmentação e forneceram resultados importantes. Mendes et al. (2014), em seu trabalho de análise do uso e ocupação da terra, fragmentação florestal e áreas de preservação permanentes no município de Cerro Azul/PR, utilizou a densidade de bordas (ED) para caracterização da paisagem. Oliveira et al. (2015), trabalharam com microcorredores ecológicos no entorno do Parque Estadual da Lapa Grande, com a métrica porcentagem da paisagem (PLAND), e foi possível realizar comparações com outros ambientes.

Para Souza et al. (2014), é possível obter um perfil do grau de fragmentação da paisagem por meio da métrica de densidade de manchas (PD), pois ela caracteriza o grau de fragmentação de determinada paisagem e ainda pode ser comparada com outras paisagens por ser relativizada. Já Pirovani et al. (2015), ressaltaram que o aumento na densidade da borda (ED) é devido as irregularidades da forma dos fragmentos após a antropização, pois são potencializadas em decorrência das atividades produtivas que acarretam a formação de novos polígonos na paisagem.

Assim como este trabalho, vários outros utilizaram métricas para quantificar a estrutura da paisagem e obtiveram resultados bastante interessantes. Leal (2016), utilizou as métricas PD, ED e PLAND para avaliar a simulação dos efeitos de padrões de desmatamento na dinâmica de uma paisagem e notou que as métricas responderam positivamente à medida que a paisagem ia sendo fragmentada pela simulação. Erica et al. (2011), trabalharam com os efeitos da mudança de escala em padrões de desmatamento na Amazônia e utilizou a métrica densidade da borda (ED) para quantificar as mudanças ocorridas, pois se trata de uma métrica interessante para diferenciação de padrões da paisagem. Já Souza et al. (2014), utilizaram a métrica proporção de ocupação da paisagem (PLAND) para quantificar a abundância proporcional de cada tipo de mancha, uma vez que essa métrica explica a composição da paisagem em que valores mais altos indicam domínio de determinada classe de uso naquela paisagem.

Esta abordagem produziu um escopo para avaliar a paisagem no entorno de florestas nacionais, o que permitiu inferir com bastante propriedade, por meio de métricas de paisagem, sobre a fragmentação nas zonas de amortecimento. Essas zonas são importantes mecanismos de proteção que devem ser monitorados e avaliados sempre que possível.

\section{CONCLUSÕES}

A criação das áreas de entorno de 0 a $10 \mathrm{~km} \mathrm{e} 10$ a $20 \mathrm{~km}$ possibilitou avaliar a fragmentação compartimentada da paisagem. Nesses compartimentos foi possível o entendimento espacial e a identificação de quais quadrantes foram mais preocupantes em relação às métricas, devido o resultado geoespacializado.

A comparação entre os dados de 2004 e 2014 tornou possível o entendimento que a dinâmica nas alterações do uso do solo não apresenta tendência que vai diminuir. Prova disso 
foram às métricas calculadas que, em pouquíssimos casos, apresentaram estabilização.

Por fim, ressalta-se a importância da regulação de fato do uso nas zonas de amortecimento que envolvem as unidades de conservação, para que não se tornem ilhas isoladas no meio de imensidões desconectadas na paisagem.

\section{REFERÊNCIAS}

ALMEIDA, C. A.; COUTINHO, A. C.; ESQUERDO, J. C. D. M.; ADAMI, M.; VENTURIERI, A.; DINIZ, C. G.; DESSAY, N.; DURIEUX, L.; GOMES, A. R. High spatial resolution land use and land cover mapping of the Brazilian Legal Amazon in 2008 using Landsat-5/TM and MODIS data. Acta Amazônica, Manaus, v. 46, n. 3, p. 291-302, 2016. DOI: http://dx.doi.org/10.1590/1809 4392201505504

BASTOS, R. Z.; FARIAS, A. L. A.; CANTO, O.; OEIRAS LEITE, E. V. Realidade e desafios da institucionalização de unidades de conservação municipais do estado do Pará, Brasil. Revista GeoAmazônia, Belém, v. 02, n. 4, p. 206220, 2014.

COUTO, P. Análise fatorial aplicada a métricas da paisagem definidas em Fragstats. Investigação Operacional, Lisboa, v. 24, n. 2, p. 109-137, 2004.

CRUZ, C.; MADUREIRA, H.; MARQUES, J. Análise espacial e estudo da fragmentação da Paisagem da Aboboreira. Revista de Geografia e Ordenamento do Território, n. 4, v. 1, p. 57-82, 2013. DOI: http://dx.doi.org/10.17127/got/2013.4.003

ERICA, A. S. FONSECA, L. M. G.; ESCADA, M. I. S.; KORTING, T, S. Efeitos da Mudança de Escala em Padrões de Desmatamento na Amazônia. Revista Brasileira de Cartografia, São José do Campos, v. 63, n. 3, p. 401-414, 2011.

FIORI, M. F.; FIORI, L. E.; NENEVÉ, M. Colonização agrícola de Rondônia e (não) obrigatoriedade de desmatamento como garantia de posse sobre a propriedade rural. Revista Novos Cadernos NAEA, Rondônia, v. 16, n. $1, \quad$ p. $9-22, \quad 2013 . \quad$ DOI: http://dx.doi.org/10.5801/ncn.v16i1.862

FRANÇOSO, R. D.; BRANDÃO, R. A. Dinâmica da paisagem no entorno da reserva natural Serra do Tombador, Norte de Goiás. Caminhos de Geografia, Uberlândia, v. 14, n. 45, p. 284-293, 2013.

INPE INSTITUTO NACIONAL DE PESQUISAS ESPACIAIS. PRODES Digital, Monitoramento da Floresta Amazônica Brasileira por Satélite. São José dos Campos, 2018. Disponível em: $<$ www.obt.inpe.br/prodesdigital/metodologia.html $>$. Acesso em: 18 de março de 2018.

LEAL, F. A. Simulação e Avaliação dos Efeitos de Padrões do Desmatamento na Dinâmica da Paisagem em São Félix do Xingu/PA. 2016. 159f. Tese (Doutorado em Ciências Florestais) - Universidade de Brasília, Brasília, 2016.

MCGARIGAL, K.; CUSHMAN, S. A.; ENE, E. FRAGSTATS v4: Spatial Pattern Analysis Program for Categorical and Continuous Maps. Amherst: Computer software program produced by the authors at the University of Massachusetts, 2012. Disponível em: < http://www.umass.edu/landeco/research/fragstats/fragstat s.html>. Acesso em: 18 de março de 2018.
MCGARIGAL, K.; MARKS, B. J. Fragstats: Spatial pattern analysis program for quantifying landscape structure. Portland: United States Deparment of Agriculture, 199. $132 \mathrm{p}$.

MENDES, B. C.; GOMES, R. A. T.; MATRICARDI, E. A. T.; FARIAS, M. F. R.; CARVALHO JÚNIOR, O. A. de; GUIMARÃES, R. F. Análise do Uso do Solo e Ocupação da Terra, Fragmentação Florestal e Áreas de Preservação Permanentes no Município de Cerro Azul-PR. Revista Espaço e Geografia, Brasília, v. 17, n. 1, p. 235-253, 2014.

OLIVEIRA, P. S.; MOREIRA, A. A.; NERY, C. V. M.; MELO, A. A. M. Microcorredores ecológicos no entorno do parque estadual da Lapa Grande. Caminhos de Geografia, Uberlândia, v. 16, n. 53, p. 189-200, 2015.

PEREIRA, B. W.; JESUIN, S. A.; MACIEL, M. N.; OLIVEIRA, F. de A.; CREÃO, L. G. C. Fragmentação da vegetação arbórea na bacia hidrográfica do Rio Apeú, Nordeste do Estado do Pará. Revista de Ciências Agrária, Belém, v. 55, n. 4, p. 297-305, 2012.

PIROVANI, D. B.; ADERBAL, G. S.; ALEXANDRE, R. S. Análise da Paisagem e Mudanças no Uso da Terra no Entorno da RPPN Cafundó, ES. Revista Cerne, Lavras, v. 21, n. 1, p. 27-35, 2015. DOI: http://dx.doi.org/10.1590/01047760201521011182

PIROVANI, D. B.; SILVA, A. G.; SANTOS, A. R.; CECÍLIO, R. A.; GLERIANI, J. M.; MARTINS, S. V. Análise espacial de fragmentos florestais na bacia do rio Itapemirim, ES. Revista Árvore, Viçosa, v. 38, n. 2, p. 271-281, 2014. DOI: http://dx.doi.org/10.1590/S010067622014000200007

RIBEIRO, M. F.; FREITAS, M. A. V.; COSTA, V. C. O desafio da gestão ambiental de zonas de amortecimento de unidades de conservação. In: Seminário Latino-Americano de Geografia Física, 6., 2010, Coimbra. Anais... Coimbra: Universidade de Coimbra, 2010. p. 01-11.

SCHMINK, M; WOOD, C. H. Conflitos sociais e a formação da Amazônia. Tradução Noemi Miyasaka Porro; Raimundo Moura. Belém: Editora UFPA, 2012. 496 p.

SILVA, I. C. V.; PAIVA, A. M. D.; SILVA, C. G.; MONTEIRO, I. P. Cenário do Desflorestamento da Amazônia Brasileira: O que dizem os Estudos. Revista Científica do Centro de Estudos em Desenvolvimento Sustentável da UNDB-CEDS, São Luís, v. 1, n. 4, p. 235 248, 2016.

SILVA, E. M. Fragmentação da Vegetação Nativa do Bioma Amazônia em Área de Fronteira Agrícola Consolidada no Norte de Mato Grosso. 2014. 65f. Dissertação (Mestrado em Ciências Florestais e Ambientais). Universidade Federal de Mato Grosso, Cuiabá, 2014

SOUZA, C. G.; ZANELLA, L.; BORÉM, R. A. T.; CARVALHO, L. M. T. de; ALVES, H. A. R.; VOLPATO, M. M. L. Análise da Fragmentação Florestal da Área de Proteção Ambiental Coqueiral, Coqueiral/MG. Revista Ciência Florestal, Santa Maria, v. 24, n. 3, p. 631-644, 2014. DOI: http://dx.doi.org/10.5902/1980509815743

VIDOLIN, G. P.; BIONDI, D.; WANDEMBRUCK, A. Análise da estrutura da paisagem de um remanescente de floresta com araucária, Paraná, Brasil. Revista Árvore, Viçosa, v. 35, n. 3, p. 515-525, 2011. DOI: http://dx.doi.org/10.1590/S0100-67622011000300014 